\title{
Towards A Comprehensive Framework For Technology Selection And Capacity Planning For Sustainable Manufacturing
}

\author{
Fahimeh Nejadi \\ Kai Cheng, Prof \\ College of Engineering, Design and Physical Sciences, \\ Uxbridge, London, UK
}

doi: 10.19044/esj.2016.v12n18p79 URL:http://dx.doi.org/10.19044/esj.2016.v12n18p79

\begin{abstract}
Technology mixed perspective, as a combination of two functions of 'Technology Selection' and 'Capacity Planning', is not usually addressed in the research literature. Yet, the importance of integrated decisions at such strategic level is evident. The overall aim of this paper is to develop a framework for combined 'technology selection' and 'capacity planning' in manufacturing sector. The approach will also incorporate the multiperspective concept of sustainability, while taking uncertainties into account.
\end{abstract}

Keywords: Technology Selection, Capacity Planning, Mathematical Programming

\section{Introduction} which are;

Today's manufacturing systems face many challenges, amongst

a) To enable integrated decisions (e.g. technology selection and capacity planning)

b) To achieve sustainable manufacturing

c) To deal with dynamism and uncertainty about the environment

Isolated decision making within manufacturing systems, especially at the strategic levels leads to conflicts, inefficiency, or at the least it could produce a sub-optimal solution. For example, two types of decisions, namely technology selection and capacity planning to have some elements in common. But they have been addressed in the literature largely in an isolated way. Integrated strategic decisions provide a better result, while it generates a larger, more complex problem to solve.

On the other hand, manufacturing firms are working in an environment that is changing on a continuous manner. For instance, demand 
for products is affected by many factors such as population, substitute products, price, competition, and so on. Such a complexity is a recipe for dynamism and uncertainty.

All the above mentioned challenges are addressed in this paper and provide solutions accordingly. This paper aims to propose a general architecture for technology selection and capacity planning simultaneously.

\section{Technology selection and capacity planning}

There are two perspectives to the technology selection problem. a) A zero-one selection where a technology is either selected or rejected, b) A combined use of different technologies, or so-called 'technology mix', which allows the split of capacity among different technologies.

Technology mix perspective is not usually addressed in widely studied scenarios in recent years. For example, the study presented in (Van de Kaa, et al., 2014) aims to analyze the data to select one dominant technology out of five available alternatives. The authors use fuzzy Analytic Hierarchy Process method to achieve their aim. (Onar, et al., 2015) concentrates on the selection of the appropriate wind energy technology. The problem is constructed as a multi-expert multi-criteria decision making problem. At (Ren \& Lutzen, 2015)VIKOR was used to evaluate and prioritize three alternative technologies out of which one technology is selected. The study by (Evans, 2013) aims to adopt an approach where both experts and non-experts can use historical decision information to support the evaluation and selection of an optimal manufacturing technology. This form of approach is based on the logic in which a decision maker would irrationally recall previous decisions to identify relationships with new problem cases. In all these types of studies, the problem is actually treated like a rather simple 0-1 decision type of scenario, where a technology is either selected or rejected. Just a fraction of these studies provide an approach with an aim to contribute to manufacturing sustainability. Yet in reality, more complex scenarios are happening.

In line with the inception of globalisation age, the emergence of global manufacturing corporations with several plants located around the world started off decades ago and is still on the rise. While some of the decisions in such a structure are made de-centrally, the importance of integrated decisions at some strategic levels, such as technology selection and capacity planning, is still evident. Such an integrated decision is supported by justifications in the areas of economies of scale and accumulated knowledge-base.

The driving force of these decisions is multi-faceted. Manufacturing technologies are subject to a continual improvement process, especially in the context of sustainable development. Technologies have a limited life- 
cycle and need to be replaced. Further, increasing demand for an existing product would require capacity expansion, while new product development could justify new technologies as well as capacity acquisition

In this paper, a model is developed with an integrated view to solve both problems 'Technology Selection' and 'Capacity Planning' simultaneously. The general scenario targeted in this research assumes that the firm's management is going to make a decision on:

"How much of capacity from which technology to acquire?"

In order to meet demands and in accordance with a number of criteria. A technology mix would enable an appropriate level of trade-off amongst conflicting criteria, such as cost, quality, and emissions. Managers might face this type of decisions in various industries and in different stages of their business, either to establish a new plant, or to expand on existing facilities, or even to replace the old technologies.

Existing studies rarely look at this combined problem of 'Technology Selection' and 'Capacity Planning' in an integrated way. (Filomena, et al., 2014) address technology selection and capacity investment under uncertainty for an electricity generation problem in a game theory-based competitive environment. The study focuses on cost evaluation against a portfolio of technologies and does not address multi-criteria nature of decisions or sustainability analysis.

\section{Sustainability perspective}

Manufacturing plays a key role in the realisation of sustainable development. Manufacturing systems make a significant contribution in creating wealth, jobs, as well as pollution. Thus the concepts of 'sustainable manufacturing' and 'sustainable technologies' are key in achieving sustainable development. When it comes to sustainable technologies, however, there is another very important dimension to consider, namely 'Technical' aspects and specifications. In fact, the major global challenges that the manufacturing sector is facing today need to be addressed in the multifaceted context of economy, society, environment and technology (ESET) (Jovane et al., 2008).

Figure1 illustrates a conceptual model of manufacturing performances from these four dimensions.

One major aspect of this research is to observe the 'Sustainability' of manufacturing systems in the course of technology selection. The proposed methodology should drive the selection of more sustainable technologies. This objective can be achieved through an optimization algorithm in which sustainability criteria are involved along with other selection criteria. In the next section, a discussion of selection criteria is presented. 


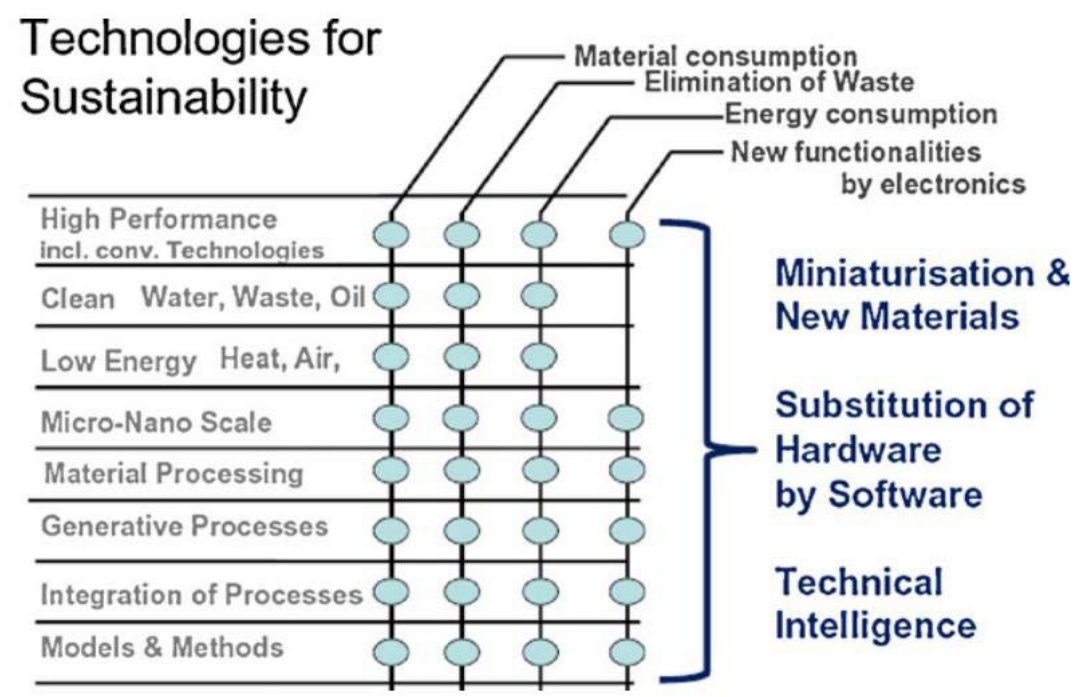

Figure 1 Technologies for sustainability (Westkamper, 2007)

\section{Technology selection criteria}

Inspired by the fundamentals of sustainability theory in the context of manufacturing, our approach to the problem 'technology selection and capacity planning' considers three criteria, namely

a) Environmental (e.g. Emissions)

b) Economic

c) Technical (e.g. quality)

These three criteria are largely in conflicting positions. Environmentally friendly technologies are usually expensive, because they may require advanced components to reduce the scale of emissions. Similar argument is true with quality and cost criteria. This gives rise to the multicriteria decision making challenge.

It is assumed that there exist regulations on controlling the environmental emissions for industries, making them keep their emissions generation at a certain level. This restriction is treated as a constraint in the proposed model.

Modelling the economic aspect of the problem is more complicated. The cost structure includes both capital and operational elements, latter of which should be considered over a time horizon. Thus, a total life-cycle costing method is proposed. The concept of 'Time Value of Money' is addressed through a discounted cash-flow method that is 'Present Value (PV)' analysis. Further, the effect of inflation rates is considered, making the proposed model more realistic. Figure 2 presents this research's triangular perspective to sustainable manufacturing. 


\section{Modelling uncertainties}

Every decision faces some uncertainties, the level of which becomes intensive where the decision has a long prospect in the future. It is assumed that the decision-making is happening in a time window through which the new circumstances might occur, e.g. prices change or criteria priorities are shifted, on the basis of which it requires the decision to be revised or adapted. Some of these uncertainties are taken into account in this research, which in turn introduce a great deal of complexity into the model.

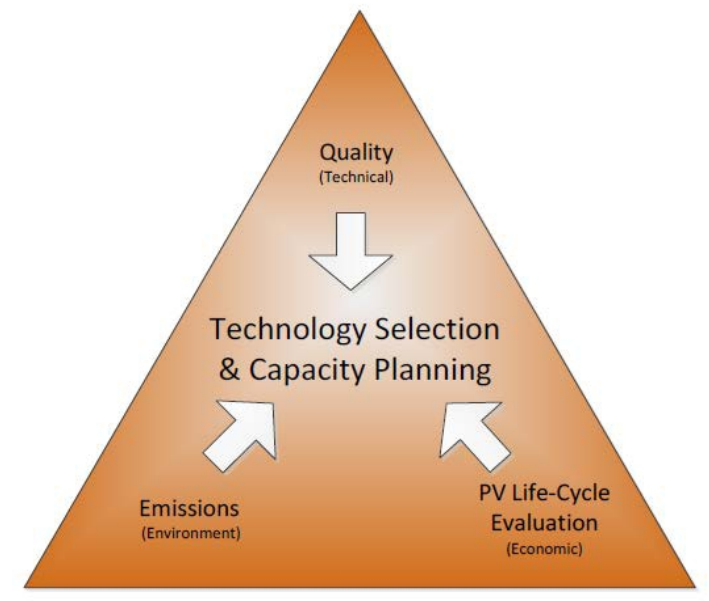

Figure 2 A triangular perspective to 'Technology Selection and Capacity Planning'

Dealing with uncertainty constitutes one of the biggest challenges in an optimisation approach. Very few studies take uncertainty into account and use a set of methods called 'Non-Deterministic or Stochastic' in general. On the other hand, the mainstream literature tends to assume that all data are certain and use the methods called 'Deterministic'. Non-deterministic models have to deal with a much larger set of data, which require high level of computational resources. Some methods use approximations to reduce the problem into a manageable size.

\section{Types of uncertainties}

There are three types of uncertainties that are addressed in this research, as follows:

Uncertainty factors with virtually no control over: Perhaps the most important uncontrollable factor associated with this type of uncertainty is;

a) Demand

This factor has a key role in capacity planning decisions. It is, however, assumed that there exists some information about future demand based on historical records or other types of sources such as expert opinions. 
Uncertainty factors with some level of flexibility: The other possibility is that although some of the parameters used in the model are beyond the user's control, they might still be influenced by the user's power, e.g. through negotiations, or give the decision maker a certain level of flexibility with the parameter value. Here are the most important ones that fall into this category:

a) Purchasing price of the technology

b) Regulatory limits

Controllable parameters: In addition, the technology selection and capacity planning problem are characterised by some parameters within the decision maker's control that could have impacts on the final results. Therefore, it is important to identify the sensitivity of the model results to the deviations on these parameters. Some of these parameters are:

a) Criteria weights

b) Rate of return ( $r r)$

c) Budget limit

\section{Proposed framework}

A framework consisting of ten major steps in four modules is proposed (figure 4). The framework puts an emphasis on facilitation of communication with users; hence 'Solution Structuring' is developed to facilitate problem solution representation.

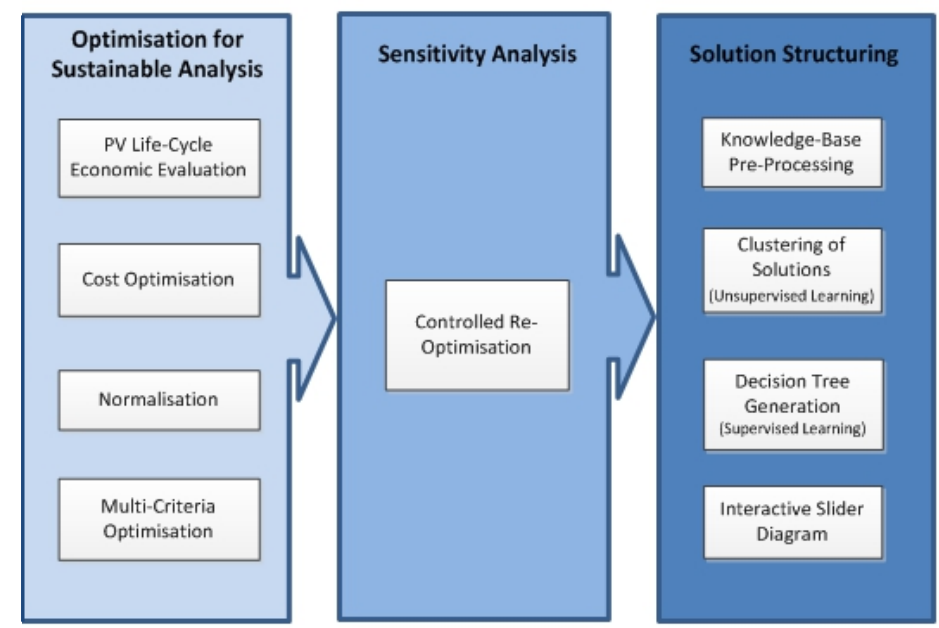

Figure 4 General architecture of an integrated optimisation approach to technology selection and capacity planning

Module 'Optimisation for Sustainable Manufacturing' addresses the optimisation of technology selection and capacity planning decisions in an integrated model. It also takes the multi-criteria aspect of sustainability in the model developed. 
'Sensitivity Analysis' module is designed to deal with the uncertainty associated with the model through scenario generations and model reoptimisations.

As a part of the 'Solution Structuring' module, the results, which constitute a large number of solution sets, are then processed using Machine Learning techniques and translated into two solution formats, namely a) decision trees, and b) interactive slider diagram.

The next sections describe the general functionalities of the above four modules as well as some algorithms developed.

\section{Optimization for sustainable analysis}

System analysis and design for achieving sustainability is a challenging task. Multi-objective decision making is fundamental to the solution approach. Analytical methods are adopted to conduct optimization of the design task.

This module consists of four steps, namely a) PV life cycle economic evaluation, b) cost optimization, c) normalization, and d) multi-criteria optimization, as described in the next sections.

\section{PV Life-Cycle economic evaluation}

Economic evaluation of design alternatives has always played a major role in decision making. Various levels of detail and evaluation methods have been used before. Due to historical and ever increasingly vital importance of the cost factor, this research attempts to present a highly detailed economic evaluation algorithm based on 'Present Value (PV)' method.

Technology investments are characterized by both initial capital spending and annual running costs. The real value of money is changed throughout time, due to the effects of 'Inflation' and 'Market Return'. PV method transforms all annual costs into their equivalent present values in a way that can be treated in the model similar to the initial capital part of an investment. Then various investments are evaluated based on their total equivalent present value. The details of the PV modelling in this research are described in the next sections:

\section{Cost structure}

Two major cost categories for each technology investment are defined in this research:

a. Capital costs (cc): Mainly includes 'purchase cost' of one unit of technology.

b. Running costs (rc): Includes five sub-categories, namely; i) materials, ii) labour, iii) energy, iv) rework, and v) maintenance. These cost 
items need to be discounted over the life of the technology (e.g. 20 years) and transformed into present value. The running cost formulae is presented below:

$M T_{i j}=\left[x_{i j} \times m t_{i j} \times\left(1+r w_{i j}\right)\right] \forall i, j$

$L_{i j}=\left[x_{i j} \times l_{i j} \times\left(1+r w_{i j}\right)\right] \forall i, j$

$E_{i j}=\left[x_{i j} \times e_{i j} \times\left(1+r w_{i j}\right)\right] \forall i, j$

$M A_{i j}=y_{i j} \times m a_{i j} \forall i, j$

Where

$\boldsymbol{M} \boldsymbol{T}_{i j}=$ cost of materials per one year production by technology $j$ for operation $i$,

$\boldsymbol{m}_{i j}=$ cost of materials per unit of product produced by technology $j$ for operation $i$,

$\boldsymbol{L}_{i j}=$ cost of labour per one year production by technology $j$ for operation $i$, $\boldsymbol{l}_{i j}=$ cost of labour per unit of product produced by technology $j$ for operation $i$,

$\boldsymbol{E}_{i j}=$ cost of energy per one year production by technology $j$ for operation $i$, $\boldsymbol{e}_{i j}=$ cost of energy per unit of product produced by technology $j$ for operation $i$,

$\boldsymbol{M} \boldsymbol{A}_{i j}=$ cost of maintenance per one year production by technology $j$ for operation $i$,

$\boldsymbol{m a}_{i j}=$ cost of maintenance per unit of technology $j$ for operation $i$,

$\boldsymbol{r} \boldsymbol{w}_{i j}=$ percentage of rework associated with technology $j$ for operation $i$,

$\boldsymbol{x}_{\boldsymbol{i}}=$ capacity volume required for technology $j$ of operation $i$;

$\boldsymbol{y}_{i j}=$ Number of units required for technology $j$ of operation $i$;

\section{The effect of inflation}

In the real world, where price inflations exist, the expenses will rise from one year to the next at the rate known as 'Inflation Rate'. In a simple word, a cost item $c$ will rise to $c(1+r i)$ next year, where $r i$ represents the rate of inflation. Therefore, the annual running costs will actually rise in real term in the form of a geometric series (Table 1). Furthermore, the inflation rates actually vary with regards to different cost items, namely materials, labor, energy, and maintenance. In this paper, the effect of inflation for each cost item is taken into account using different inflation rates.

Table-1 Geometric series representing the effect of inflation

\begin{tabular}{|c|c|c|c|c|c|}
\hline Year & 1 & 2 & 3 & $\ldots$ & t \\
\hline Cost & rc & rc & rc & $\ldots$ & rc \\
\hline Inflated Cost & rc x (1+ri) & rc x (1+ri) $)^{2}$ & rc x (1+ri $)^{3}$ & & rc x (1+ri) ${ }^{\mathrm{t}}$ \\
\hline
\end{tabular}

\section{The effect of market return}

Market return is a profit on an investment, also called return on investment (ROI). It is a measure of investment performance. The effect of 
market return is involved when dealing with cash flows over time. Market return is represented by the Rate of Return (rr) in a sense that every pound invested at time zero should grow over time at a rate rr when used in a business. In other words, every pound spent at time one would be worth $1 /(1+\mathrm{rr})$ at time zero. The equivalence value of running costs at time zero when considering the effect of market return can be shown in Table 2.

Table 2 the effect of market returne on investment rate on annual running costs

\begin{tabular}{|c|c|c|c|c|c|}
\hline Year & 1 & 2 & 3 & $\ldots$ & $\mathrm{t}$ \\
\hline Cost & rc & rc & rc & $\ldots$ & rc \\
\hline $\begin{array}{c}\text { Equivalent } \\
\text { Cost at year 0 } \\
\text { (effect of } \\
\text { market return) }\end{array}$ & $\boldsymbol{r c} /(\mathbf{1}+\boldsymbol{r} \boldsymbol{r})$ & $\boldsymbol{r c} /(\mathbf{1}+\boldsymbol{r} \boldsymbol{r})^{2}$ & $\boldsymbol{r c} /(\mathbf{1}+\boldsymbol{r} \boldsymbol{r})^{3}$ & & $\boldsymbol{r c} /(\mathbf{1}+\boldsymbol{r} \boldsymbol{r})^{\mathrm{t}}$ \\
\hline
\end{tabular}

\section{Present Value (PV) model}

The PV model of annual running cost $r c$ when considering the combined effects of inflation and market return can be shown in the form of a new geometric series as follows:

$\mathrm{Or}$

$$
\frac{r c(1+r i)}{(1+r r)}, \frac{r c(1+r i)^{2}}{(1+r r)^{2}}, \frac{r c(1+r i)^{3}}{(1+r r)^{3}}, \ldots
$$

$\boldsymbol{r c} \times d \boldsymbol{r}, \boldsymbol{r c} \times d r^{2}, r c \times d r^{3}, \ldots$

Where $d r\left(=\frac{(1+r i)}{(1+r r)}\right)$ represents the combined discounting effects of both inflation rate and return on investment.

The summation of the series terms, which represents the equivalent present value of all running costs of the technology $j$ for operation $i$, is calculated using the following formulae:

$$
\text { Running Cost Present Value }=r c_{i j} \times d r \times\left(\frac{1-d r^{t_{i j}}}{1-d r}\right)
$$

Where $t_{i j}$ refers to the life period of the technology $j$ for operation $i$. Finally, the total PV of each technology investment, including both capital and running costs, can be calculated as follows:

$$
\begin{aligned}
P V_{i j}=c c_{i j}+ & {\left[M T_{i j} \times d r \times\left(\frac{1-d r^{t_{i j}}}{1-d r}\right)+\left[L_{i j} \times d r \times\left(\frac{1-d r^{t_{i j}}}{1-d r}\right)+\left[E_{i j}\right.\right.\right.} \\
& \times d r \times\left(\frac{1-d r^{t_{i j}}}{1-d r}\right)+\left[M A_{i j} \times d r \times\left(\frac{1-d r^{t_{i j}}}{1-d r}\right)\right]
\end{aligned}
$$

\section{Cost Optimization}

This part of the proposed framework is particularly responsible for finding the total cost goal for the purpose of normalization as described later in the next section. The cost optimization model is similar to the main model 
assuming there is only one criterion to consider, namely cost. A Linear Programming (LP) model is developed to solve this problem.

\section{Normalization}

One of the first steps in a multi-criteria approach is to normalize the effects of various criteria. In normalization, impact potentials and resource consumptions are expressed on a common scale by relating them to a common reference, to enable a comparable assessment across impact categories (Wenzel, et al., 2001) .All potential impacts are converted into the same units to facilitate a systematic comparison. To compare different impact potentials, an evaluation should be based on the seriousness of the impact, which is assessed by a set of weighting factors.

All the three criteria mentioned earlier in section 4 require normalization transformations in order to enable a comparable evaluation of the various scenarios. Common normalization algorithms convert various scales into a unique scale for all the criteria scores. 'Normalization algorithm by comparison with the best value' method is adopted in this research. This method can be formulated in two ways, as presented below, assuming that the criterion is to be minimized.

\section{Normalised Score}

$=$

$1-$

criteria value for a specific alternative-Min. criteria value across all the alternatives

Min. criteria value across all the alternatives

Normalised Score $=\frac{\text { Min. } \text { criteria value across all the alternatives }}{\text { criteria value for a specific alternative }}$

The first formula was, however, preferred in this research due to the fact that the second one turned the linear model into a non-linear one, which compromises on the efficiency and effectiveness of the solution.

Normalization algorithm requires a target (or best) value for each criterion. In the current research, the best criteria value across different technologies for a specific operation is used as the target value for normalization calculations. The normalized transformation of the best value is set to ' 1 ' based on the above formula.

There are, however, some complications involving the technology mix nature of the problem. Technology mix makes both optimisation and normalisation algorithms more complicated, because the number of alternatives is numerous. Each alternative could involve a mixed use of several technologies per operation. Under such circumstances, calculations in normalisation algorithms are carried out per each technology mix rather than per each individual technology.

\section{Normalised environmental impact measure:}


It is calculated based on the following formulae, as presented above.

$$
N E_{i}=1-\frac{\sum_{j}\left(e_{i j} \times x_{i j}\right)-\operatorname{Min}_{j}\left(e_{i j}\right) \times D_{i}}{\operatorname{Min}_{j}\left(e_{i j}\right) \times D_{i}} \quad \forall i
$$

Where $N E_{i}$ denotes the normalised environmental impact value associated with operation $i\left(N E_{i} \in\{0,1\}\right.$ with 1 being the least environmental impact), $e_{i j}$ denotes raw environmental impact value associated with technology $j$ for operation $i, x_{i j}$ denotes the capacity acquisition of technology $j$ for operation $i$, and $D_{i}$ denotes the level of demand for operation $i$. It is important to note that the environmental impacts of technologies are compared for each operation rather than across all operations. Therefore, $\operatorname{Min}_{j}\left(e_{i j}\right)$ is referred to the minimum environmental impact value across technologies available for operation $j$.

\section{Normalised technical measure}

Assuming that technical scores are allocated in a way that best scenario will get a lower score, the normalised score, similar to the environmental measure, is calculated based on the following formulae: $N T_{i}=1-\frac{\sum_{j}\left(t_{i j} \times x_{i j}\right)-\operatorname{Min}_{j}\left(t_{i j}\right) \times D_{i}}{\operatorname{Min}_{j}\left(t_{i j}\right) \times D_{i}} \quad \forall i$

Where $N T i$ denotes the normalised technical (quality) value associated with operation $i$ (NTiE\{0,1\} with 1 being the highest technical index), and $t_{i j}$ denotes raw technical value -such as quality rejection rate associated with technology $j$ for operation $i$. It is important to note that $t_{i j}$ represents a variable that is to be minimised.

\section{Normalised economic measure}

As for the economic measure, the best value cannot be obtained as straightforward as it was with the other two criteria. This is due to the fact that the cost structure of each technology is more complicated and consists of several elements, as described earlier in section 6.1.1. Therefore the minimum total cost value needs to be obtained. This is carried out using an auxiliary model called 'Cost Optimisation', as discussed earlier in 6.1.2. The cost optimisation is carried out through a separate mathematical model that finds the minimum total cost across all the operations. Therefore, the normalised economic score is calculated based on the following formulae;

$$
N C=1-\frac{t c-t c *}{t c *}
$$

Where $N C$ refers to the normalized economic value $(N C \leq 1$ with 1 being the lowest total cost), $t c$ refers to the total cost associated with each technology combination scenario across all operations, and $t c^{*}$ refers to the minimum total cost across all operations. 


\section{Multi-Criteria optimization}

This element of the research framework is responsible for the actual multi-criteria optimization of technology selection and capacity planning for a sustainable manufacturing. The problem addressed in this research is characterized by a number of complexities, namely:

- The 'technology mix' approach to the problem. This makes optimization algorithms more complicated compared to the situation where there are only two states; 'selection' or 'no selection'. Instead, a numerous number of combinatorial scenarios need to be considered in this research.

- $\quad$ The model is to work under uncertainty. Problem-solving approaches under uncertainty face a huge complication, dealing with uncertain data.

- Multi-criteria nature of sustainability perspective, where different types of criteria (both quantitative and qualitative) are taken into account.

The main approach used in this framework is 'Mathematical Programming', which is a general term for a suit of methods. More specifically, a Goal Mixed Integer/Linear Programming method is used, where Goal Programming model is responsible for multi-criteria optimisation and the Mixed Integer/Linear Programming model deals with an integrated optimisation of technology selection and capacity planning. Further details about the Multi-Criteria Optimisation module can be found in future publications.

\section{Sensitivity Analysis}

Decisions are made in a highly uncertain environment. This means that the results might not be valid by the time the decisions are to be implemented. Also, the decision-making might be happening in a time window through which the new circumstances might occur, on the basis of which it requires the decision to be revised or adapted. There are several ways to deal with uncertainty. A related field is sensitivity analysis. With sensitivity analysis one can ascertain the impact of the uncertainty with respect to the parameters' values on the quality of the optimum solution. Uncertainty analysis and sensitivity analysis are essential parts of analyses for complex systems.

The proposed approach to high variability situations in this research is to predict possible scenarios in advance and pre-plan for each. The focus of this research is on sampling-based sensitivity analysis, which is an effective and widely used approach (Helton, 2008).

There is a very important property of the Linear Programming (LP) models that is called 'Duality'. Knowledge of the duality provides interesting economic and sensitivity analysis insights to the problem. The optimization model developed in this research is, however, essentially of a mixed Integer/Linear Programming type, which does not allow the applicability of 
duality. Therefore, a controlled re-optimization approach was adopted where the original model is re-optimized for a set of sampled input data. Each reoptimization episode is characterized by one set of input data against one set of output results. The re - optimization module uses the same method as used in the original optimization step. A software tool is designed to control the re-optimization process in an efficient way. Further details on this module are presented in future publications.

\section{Solution structuring}

Sensitivity analysis module provides rich pieces of knowledge for decision makers in a form of scenario-solution pairs in the scale of hundreds or perhaps thousands. Such a massive knowledge, however, needs to be structured in an abstract way, yet scientifically sound to represent the knowledge originally generated.

This research adopts 'Machine Learning' approach to the solution structuring stage of the framework. More specifically, two types of knowledge structuring are suggested, including:

I. Decision tree

II. Interactive slider diagram

These representation schemes provide decision-maker with a variety of methods to structure the solution set. Decision tree is a widely-used and effective knowledge representation scheme. 'Machine Learning' in general and a combination of 'Clustering' and 'Classification' algorithms are applied in this research to generate the best decision tree.

\section{Conclusion}

In this paper, a model is developed with an integrated view to solve two problems 'Technology Selection' and 'Capacity Planning' simultaneously. A 'technology mix' decision is allowed, which enables an appropriate level of trade-off amongst conflicting criteria, such as cost, quality, and emissions. A framework consisting of nine major steps in three modules is proposed, namely a) Multi-Criteria Optimisation, b) Sensitivity Analysis, and c) Solution Structuring.

One major aspect of this research is to observe the 'Sustainability' of manufacturing systems in the course of technology selection. The proposed methodology should drive the selection of more sustainable technologies. This objective is achieved through an optimisation algorithm in which sustainability criteria are involved along with other selection criteria. Three criteria are considered, including a) Environmental (e.g. Emissions), b) Economic, and c) Technical (e.g. quality).

In summary, the main characteristics of the proposed framework are: 
1. An integration of technology selection and capacity planning functions.

2. Tackling uncertainty

3. $\quad$ Addressing multi-operation problems

4. Considering the effects of inflation and market return

5. Total life-cycle economic evaluation

6. Sustainability perspectives

7. Decision tree-based solution structuring

8. Technological constraints (incompatible technologies)

\section{References:}

Evans, L., 2013. Experience-based decision support methodology for manufacturing technology selection. A fuzzy-decision- tree mining approach (Doctoral dissertation, university of Nottingham).

Filomena, T., Campos-Nanez, E. \& Duffy, M., 2014. Technology selection and capacity investment under uncertainty. European Journal of Operational Research, 232(1), pp. 125-136.

J.C. Helton, Uncertainty and sensitivity analyss for models of complex systems, in Computational Methods in Transport: Verification and Validation, T.J. Barth, M. Griebel, D.E.

Keyes, R.M. Nieminen, D. Roose, and T. Schlick, eds., Springer, Berlin, Heidelberg, 2008,pp. 207-228.

Jovane, F. et al., 2008. The incoming global technological and industrial revolution towards competitive sustainable manufacturing. CIRP Annals Manufacturing Technology, 57(2), pp. 641-659.

Kiritsis D, 2007, Sustainable Manufacturing, In IMS Conf. Proc. 'Strategies for Global Manufacturing A European View’, 15-16 November 2007, ETHZ, Zürich.

Onar, S. C., Oztaysi, B., Otay, i. \& Kahraman, C., 2015. Multi-expert wind energy technology selection using interval-valued intuitionstic fuzzt sets. Energy, 90, pp. 274-285.

Ren, J. \& Lutzen, M., 2015. Fuzzy multi-criteria decision-making method for technology selection for emissions reduction from shipping under uncertainties. Transportation research Part D: Transport and Environment, 40, pp. 43-60.

Van de Kaa, G., Rezaei , J., Kamp, L. \& Winter, A. d., 2014. Photovoltaic technology selection: A fuzzy MCDM approach. Renewable and Sustainable Energy Reviews, 32, pp. 662-670.

Westkamper, E., 2007. Sustainable manufacturing. Porto, Manufacture 2007 Conference. 